\title{
Self-rated Accuracy of Rating of Perceived Exertion-based Load Prescription in Powerlifters
}

Eric R. Helms a , Scott R. Brown ${ }^{\text {a }}$, Matt R. Cross ${ }^{\text {a }}$, Adam Storey ${ }^{\text {a }}$, John Cronin ${ }^{\text {a, b }}$, Michael C. Zourdos ${ }^{\mathrm{c}}$

${ }^{a}$ Sport Performance Research Institute New Zealand (SPRINZ), Auckland University of Technology, Auckland, New Zealand.

${ }^{\mathrm{b}}$ School of Exercise, Biomedical and Health Sciences, Edith Cowan University, Perth, Australia.

${ }^{c}$ Department of Exercise Science and Health Promotion, Muscle Physiology Laboratory, Florida Atlantic University, Boca Raton, FL.

Eric Helms

Sports Performance Research Institute New Zealand (SPRINZ), AUT University

AUT Millennium Institute

17 Antares Place, Mairangi Bay, Auckland 0632, New Zealand

Tel: (64) 021638466

E-mail: eric.helms@aut.ac.nz 
Prescribing Load by RPE 1

\section{$1 \quad$ Abstract}

2 This study assessed male $(n=9)$ and female $(n=3)$ powerlifters' (18-49yrs) ability to select loads using the repetitions in reserve (RIR)-based rating of perceived exertion (RPE) scale for a single

4 set for squat, bench press and deadlift. Subjects trained $3 x / w k$. for $3 w k s$. on non-consecutive 5 days in the weekly order of hypertrophy (8-repetitions at 8 RPE), power (2-repetitions at 8 RPE), 6 and strength (3-repetitions at 9 RPE), using subject-selected loads intended to match the target 7 RPE. Bench press and squat were performed every session and deadlift during strength and 8 power only. Mean absolute RPE differences (|reported RPE - target RPE|) ranged from 0.2290.44 , with a mean of $0.33 \pm 0.28$ RPE. There were no significant RPE differences within-lifts 10 between sessions for squat or deadlift. However, bench press was closer to the target RPE for 11 strength $(0.15 \pm 0.42 \mathrm{RPE})$ vs. power $(-0.21 \pm 0.35 \mathrm{RPE}, \mathrm{p}=0.05)$. There were no significant differences within-session between lifts for power and strength. However, bench press was closer $(0.14 \pm 0.44 \mathrm{RPE})$ to the target RPE than squat $(-0.19 \pm 0.21 \mathrm{RPE})$ during hypertrophy $(\mathrm{p}=0.02)$. Squat power was closer to the target RPE in week $3(0.08 \pm 0.29 \mathrm{RPE})$ vs $1(-0.46 \pm 0.69 \mathrm{RPE}$, $\mathrm{p}=0.03$ ). It seems powerlifters can accurately select loads to reach a prescribed RPE. However, accuracy for 8-repetition sets at 8 RPE may be better for bench press compared to squat. Rating squat power-type training may take 3 wks. to reach peak accuracy. Finally, bench press RPE accuracy appears better closer rather than further from failure (i.e. 3-repetition 9 RPE sets vs. 2repetition 8 RPE sets).

\section{Key Words: resistance training, autoregulation, powerlifting, periodization, load} prescription. 


\section{INTRODUCTION}

It has been reported that there is a wide disparity of repetitions allowed at various percentages of one-repetition maximum (1RM) among individuals (7) and large fluctuations of resistance training performance based upon daily readiness $(5,9)$. Thus, the repetitions in reserve (RIR) -based rating of perceived exertion (RPE) scale (11) was designed to autoregulate training load based upon daily readiness (3), and equate effort per set across individuals. Therefore, instead of prescribing a number of repetitions at a particular percentage of $1 \mathrm{RM}$, a number of repetitions can be prescribed with a target RPE i.e. 8 repetitions at an 8 RPE (2 RIR).

The RIR-based scale has specific utility because less than maximal Borg RPE ratings are sometimes given by subjects even when performing sets to failure (2); in contrast the RIR-based scale seems especially accurate when training near to failure. Importantly, it was demonstrated that trained males and females estimated RIR accurately (an RIR error of $<1$ ) when performing sets 0-3 repetitions from failure with a predetermined load (1) however, RIR was less accurate when performing sets further from failure $(1,2)$. Additionally, RPE/RIR accuracy has been shown to improve with training experience (3). However, there is no study examining the accuracy of self-selected loads (i.e. no predetermined load) to comply with the desired RPE.

Therefore, the aim of this study was to assess nationally qualified male and female powerlifters' ability to accurately select loads resulting in a target RIR-based RPE for a single set in the squat, bench press and deadlift on hypertrophy-, power- and strength-type sessions over three weeks. We hypothesized accuracy would be the same between lifts, as similar RPE has been previously reported among the powerlifts at $1 \mathrm{RM}$ in powerlifters (4). Additionally, we hypothesized accuracy during lower RPE hypertrophy and power sessions (target RPE $=8$ ) would be less than the higher RPE strength sessions (target RPE $=9$ ). Finally, we postulated 
accuracy would improve over three weeks as subjects gained familiarity with this training approach.

\section{METHODS}

\section{Experimental Approach to the Problem}

Competitive powerlifters performed the squat and bench press $3 \mathrm{x} / \mathrm{wk}$. and the deadlift $2 \mathrm{x} / \mathrm{wk}$. (only strength and power sessions) for three weeks in a daily undulating format. Weekly session order was hypertrophy-, power-, then strength-type on non-consecutive days (i.e. Mon., Wed., Fri.) (10). Immediately before an initial 1RM testing session, which occurred 72 hours prior to the first training session, the RIR-based RPE scale was shown to each participant and described in detail (11). The scale was shown to subjects following all warm-up and working sets during testing.

During training, an RPE target was provided for a specific number of repetitions on the initial working set for each lift; thus, subjects self-selected the load they believed would result in the target RPE. Only the load for the initial set was selected by the participants (subsequent sets were adjusted based on post-set RPE score). Therefore, to determine RPE accuracy, differences between the target RPE and actual RPE after the initial set for each exercise were analyzed.

\section{Subjects}

Fourteen powerlifters were recruited and twelve completed the protocol; nine males (height $1.71 \pm 0.06 \mathrm{~m}$; body mass $81.9 \pm 12.5 \mathrm{~kg}$ ) and three females (height $1.62 \pm 0.08 \mathrm{~m}$; body mass $59.0 \pm 5.8 \mathrm{~kg}$ ). Two (male: $\mathrm{n}=2$ ) dropped out due to injury or inability to complete all sessions. Inclusion criteria was as follows: 1) minimum resistance training experience $1 \mathrm{yr} ; 2$ ) meeting the New Zealand powerlifting national qualifying strength requirements in prior competition (within one year) or during testing (6);3) compliance with the banned substance list 
of the International Powerlifting Federation (IPF) (8); 4) be between 18-49yrs old; and, 5) be free from injury/illness. All subjects were informed of potential risks and signed an informed consent document prior to participation (University ethics approval number 15/06).

\section{Procedures}

Rating of Perceived Exertion. The RIR-based RPE scale (i.e. RPE scores which correspond to RIR) (Figure 1) (11) was used throughout the study. The scale was shown and explained to each subject in the same exact manner prior to pre-testing and was shown to each subject following all warm-up and working sets.

\section{INSERT FIGURE 1 HERE}

Training Protocol. Three weeks of training were completed with a program similar to a previous undulating powerlifting protocol (10) in that each session had a specific goal: Monday: "hypertrophy" (8-repetitions at an 8 RPE), Wednesday: "power" (2-repetitions at an 8 RPE) and Friday: "strength" (3-repetitions at a 9 RPE). The squat and bench press were performed in all sessions, while deadlift was performed only on power and strength sessions to minimize injury risk and to comply with common powerlifting methods. Subjects were trained in the "offseason", i.e. they were not in the immediate pre-competition training phase. Subjects were instructed to not modify their nutrition or nutritional supplementation during the study and all sessions for each individual were carried out at the same time of day whenever possible. In each session, lifts were performed in competition order: squat, then bench press and then deadlift (if performed), following a dynamic warm-up and warm-up sets. There was a 5-minute rest period after the completion of a lift before the next was initiated. After each warm-up set RPE was obtained, and after all warm-up sets the subject was informed of the repetition and RPE target for the day. Following warm-up sets, a 3-minute rest was administered, then subjects performed the working 
set with a self-selected load with the goal of meeting the target repetitions and RPE. Consultation of prior session data was allowed to assist load selection.

\section{Statistical Analyses}

To quantify the directionality of error, 'RPE difference' (RPEDIFF) of target versus reported RPE was recorded (reported RPE score - RPE target). Thus, negative numbers represent 'undershooting' target RPE, while positive represent an 'overshoot'. Since RPE corresponds to RIR, missed repetitions counted as a full RPE score overshoot. This data is displayed in Figure 2. To display 'absolute accuracy', the mean absolute RPEDIFF (negative sign excluded for RPE undershoot) for each lift for each session was calculated. Thus, absolute RPEDIFF values were averaged for squat hypertrophy week 1,2 and 3, bench press power week 1, 2 and 3, deadlift strength week 1, 2, and 3 etc., for each subject. This data is displayed in Table 1.

Non-parametric statistical comparisons were made using RPEDIFF values (sign included). Both RPEDIFF over and undershoot values were averaged to generate means so that differences in directionality (under and overshooting) of accuracy could be assessed.

Comparisons were made from each week, for each lift, for the same training session compared to the other lifts (i.e. squat hypertrophy vs. bench press hypertrophy). Additionally, comparisons were made within the same lift, between training sessions (i.e. bench press hypertrophy vs. bench press power vs. bench press strength). Finally, comparisons were made between weeks for the same lift, during the same session to assess the effect of time (i.e. deadlift power week $1 \mathrm{vs}$ deadlift power week 2 vs deadlift power week 3).

A Friedman test with an alpha set at 0.05 was used for comparisons between two variables (i.e. squat and bench press comparisons on hypertrophy sessions). When three variables 
were compared (i.e. hypertrophy vs. power vs. strength for the bench press), a Friedman test followed by a post hoc Wilcoxon signed rank test was used. A Bonferonni correction was used for three variable comparisons. Analysis was performed using a statistical software package (IBM SPSS Statistics 21, SPSS Inc., Chicago, IL).

\section{RESULTS}

\section{RPE 'Under' and 'Overshoot'}

Figure 2 displays RPEDIFF without the sign dropped to demonstrate RPE 'over' and 'undershoot' throughout the study with ' $\mathrm{X}$ ' values displaying RPEDIFF among individual subjects (darker x's signify a greater number of subjects with the same RPEDIFF).

\section{INSERT FIGURE 2 HERE}

\section{Absolute RPEDIFF Scores}

Table 1 displays RPEDIFF values, with the sign dropped, for the group and individuals to show 'absolute accuracy'.

\section{INSERT TABLE 1 HERE}

\section{Within-lift RPEDIFF Comparisons between Sessions}

Squat RPEDIFF comparisons between hypertrophy (-0.19 $\pm 0.21 \mathrm{RPE})$, power $(-0.10 \pm$ $0.45 \mathrm{RPE})$ and strength $(0.01 \pm 0.37 \mathrm{RPE})$ sessions were not significantly different (raw $\mathrm{p}=0.07$ to 0.76 ; Bonferroni corrected $\mathrm{p}=0.22$ to 0.99$)$. Bench press RPEDIFF for hypertrophy $(0.14 \pm$ $0.44 \mathrm{RPE})$ was closer to the RPE target compared to power $(-0.21 \pm 0.35 \mathrm{RPE})$, but this difference only approached significance after ad hoc testing (raw $\mathrm{p}=0.03$; Bonferroni corrected $\mathrm{p}=0.10)$. Bench press RPEDIFF for strength $(0.15 \pm 0.42 \mathrm{RPE})$ was significantly closer than power to the target RPE (raw $\mathrm{p}=0.02$; Bonferroni corrected $\mathrm{p}=0.05$ ). Bench press RPEDIFF 
141 for strength vs. hypertrophy were not significantly different (raw $\mathrm{p}=0.94$; Bonferroni corrected $\mathrm{p}=0.99)$. Finally, deadlift RPEDIFF for strength $(0.04 \pm 0.41 \mathrm{RPE})$ was not significantly different than power $(-0.08 \pm 0.23$ RPE, $p=0.16)$.

\section{Within-session RPEDIFF Comparisons between Lifts}

Bench press RPEDIFF was closer to the RPE target compared to squat on hypertrophy sessions $(\mathrm{p}=0.02)$. All comparisons of RPE differences during power sessions among the lifts were non-significant (raw $p=0.17$ to 0.72 ; Bonferroni corrected $p=0.50$ to 0.99 ). Likewise, all comparisons of RPE differences during strength sessions among the lifts were non-significant (raw $\mathrm{p}=0.58$ to 0.81 ; Bonferroni corrected $\mathrm{p}=0.99$ ).

\section{RPEDIFF over Time}

To assess whether the accuracy of load selection to reach RPE targets changed over time, RPEDIFF was assessed across weeks. There was a difference approaching statistical significance indicating that week-3 $(-0.04 \pm 0.26 \mathrm{RPE})$ vs. week-1 $(-0.33 \pm 0.39 \mathrm{RPE})$ accuracy may have improved during squat hypertrophy sessions (raw $\mathrm{p}=0.04$; Bonferroni corrected $\mathrm{p}=0.11$ ). Likewise, a difference approaching significance indicated that week-2 $(0.08 \pm 0.67 \mathrm{RPE})$ vs. week-1 (-0.46 \pm 0.69 RPE) accuracy may have improved for squat in power sessions (raw $\mathrm{p}=$ 0.03; Bonferroni corrected $\mathrm{p}=0.09)$. Week-3 RPEDIFF for squat in power sessions $(0.08 \pm 0.29$ RPE) was significantly more accurate vs. week-1 (raw $p=0.01$; Bonferroni corrected $p=0.03$ ). correction. 
Prescribing Load by RPE 5

\section{DISCUSSION}

The purpose of this investigation was to assess if powerlifters could accurately self-select loads corresponding to a target RPE and number of repetitions. Our first hypothesis, that RPEDIFF would be similar between lifts, was mostly supported in that the comparisons were non-significant during strength and power sessions. However, RPE scores for bench press were closer to the target RPE than squat during hypertrophy sessions $(\mathrm{p}=0.02)$. Our second hypothesis, that RPE scores during strength sessions would be closer to the target (RPE 9) than hypertrophy and power sessions (RPE 8), was mostly unsupported as the accuracy of strength session RPE was only statistically superior to power for the bench press (Bonferroni corrected $\mathrm{p}$ $=0.05)$. Finally, our premise that reported RPE would be closer to the target over time as accuracy improved, was only true for squat hypertrophy sessions in week three vs. week one (Bonferroni corrected $\mathrm{p}=0.03$ ).

A potential explanation for why RPE was closer to the target for bench press compared to squat during hypertrophy sessions, is that squats arguably require more technical skill and generate more systemic fatigue due to the amount of musculature involved. Thus, there is a greater chance of a technique error, causing greater RPE variability, with high repetition squats compared to the bench press. To reconcile our second hypothesis being unsupported, Hackett and colleagues recently reported RIR to be accurately estimated when repetitions were within 03 of failure $(1,2)$, which would encompass all present target RPEs (8-9 RPE = 1-2 RIR). Regarding our final hypothesis of improvement over time with RPE, statistically there was only an improvement in the squat during power sessions (week 2 vs. 1 , Bonferroni corrected $p=0.09$; week 3 vs. 1, Bonferroni corrected $p=0.03$ ). Although, there was also a trend for improvement during squat hypertrophy sessions (week 3 vs. 1 , Bonferroni corrected $\mathrm{p}=0.11$ ). As previously 
stated, the squat arguably requires the most technical proficiency to perform. This, combined with lower target RPE on power and hypertrophy sessions relative to strength sessions, may be why a learning effect was observed only when a lower RPE was combined with the most complex lift. However, it can be observed from the data in Figure 2 (panels A, B and C) that the spread of RPE scores tightened around the target as the lifters progressed from weeks 1 to 3 , with the exception of two outlier performances in week 3 . Additionally, it is possible that 3 weeks is not a long enough time frame to demonstrate improvements in RPE accuracy.

Overall, accurate loads were selected to reach the target RPE. Even when extending absolute RPEDIFF two SDs from the mean, values were $\sim 1$ RPE from the target on average (Table 1). However, limitations do exist: sets were not performed to failure (except in error when exceeding the target RPE) thus, whether RPE scores represented 'true' RIR is unknown; however, it has previously been reported that intra-set RIR ratings were accurate when sets were close to failure $(1,2)$. Finally, accuracy was only examined in one set, thus future research should examine the ability to meet an RPE target with a self-selected load on subsequent sets once fatigue (neuromuscular and metabolic) is present.

\section{PRACTICAL APPLICATIONS}

Powerlifters can select loads to reach a self-rated target RPE with precision after a familiarization session explaining and using the RPE scale. However, achieving peak accuracy levels for the squat at RPE targets below 9 may require at least three weeks. Additionally, it seems that RPE ratings for the bench press are more accurate when performing low repetition sets closer to failure, and powerlifters are slightly better at selecting a load for an RPE target with high repetitions (8-repetitions at RPE 8) in the bench press vs. squat. However, the between lift 
difference magnitude is low in that on average, powerlifters had an absolute error of $0.33 \mathrm{RPE}$,

211 with a mean range of 0.22-0.44 RPE (Table 1). Thus, practical differences in accuracy between

212 lifts and sessions may be inconsequential. Practically, we recommend that RPE targets can be

213 used for load prescription in powerlifters however, it is unknown if untrained lifters can

214 effectively self-select a target RPE load.

215 Table and Figure Legend:

216 Table 1. 3-week average absolute RPEDIFF values.

217 Figure 1. RIR-based RPE scale

218

Figure 2. RPEDIFF values of powerlifters performing the squat, bench press and deadlift over 3 weeks.

\section{References}

1. Hackett DA, Cobley S, Davies T, Michael S, and Halaki M. Accuracy in estimating repetitions to failure during resistance exercise. J Strength Cond Res Publish Ahead of Print, 2016.

2. Hackett DA, Johnson NA, Halaki M, and Chow CM. A novel scale to assess resistanceexercise effort. Journal of sports sciences 30: 1405-1413, 2012.

3. Helms ER, Cronin J, Storey A, and Zourdos MC. Application of the Repetitions in Reserve-Based Rating of Perceived Exertion Scale for Resistance Training. Strength Cond J 38: 42-49, 2016.

4. Helms ER, Storey A, Cross MR, Brown SR, Lenetsky S, Ramsay H, Dillen C, and Zourdos MC. RPE and Velocity Relationships for the Back Squat, Bench Press, and Deadlift in Powerlifters. J Strength Cond Res 31: 292-297, 2017.

5. McNamara JM and Stearne DJ. Flexible nonlinear periodization in a beginner college weight training class. J Strength Cond Res 24: 2012-2017, 2010.

6. $\quad$ http://www.nzpowerlifting.co.nz/. Accessed Mar 6/2015.

7. Richens B and Cleather DJ. The relationship between the number of repetitions performed at given intensities is different in endurance and strength trained athletes. Biology of Sport 31: 157-161, 2014.

8. $\quad$ https://wada-main-prod.s3.amazonaws.com/resources/files/wada-2016prohibited-list-summary-of-modifications-en.pdf. 
9. Zourdos MC, Dolan C, Quiles JM, Klemp A, Jo E, Loenneke JP, Blanco R, and Whitehurst M. Efficacy of daily one-repetition maximum training in well-trained powerlifters and weightlifters: a case series. Nutr Hosp 33: 437-443, 2015.

10. Zourdos MC, Jo E, Khamoui AV, Lee SR, Park BS, Ormsbee MJ, Panton LB, Contreras RJ, and Kim JS. Modified Daily Undulating Periodization Model Produces Greater Performance Than a Traditional Configuration in Powerlifters. Journal of strength and conditioning research 30: 784-791, 2016.

11. Zourdos MC, Klemp A, Dolan C, Quiles JM, Schau KA, Jo E, Helms E, Esgro B, 
Table 1. 3-week average absolute RPEDIFF values

\begin{tabular}{|c|c|c|c|c|c|c|c|c|c|}
\hline $\begin{array}{l}\text { Subject } \\
\text { number }\end{array}$ & $\begin{array}{c}\text { Squat } \\
\text { hypertrophy }\end{array}$ & $\begin{array}{l}\text { Squat } \\
\text { power }\end{array}$ & $\begin{array}{c}\text { Squat } \\
\text { strength }\end{array}$ & $\begin{array}{l}\text { Bench press } \\
\text { hypertrophy }\end{array}$ & $\begin{array}{l}\text { Bench } \\
\text { press } \\
\text { power }\end{array}$ & $\begin{array}{c}\text { Bench } \\
\text { press } \\
\text { strength }\end{array}$ & $\begin{array}{c}\text { Deadlift } \\
\text { power }\end{array}$ & $\begin{array}{l}\text { Deadlift } \\
\text { strength }\end{array}$ & $\begin{array}{c}\text { Combined } \\
\text { averages }\end{array}$ \\
\hline 1 & 0.33 & 0.00 & 0.17 & 0.50 & 0.33 & 0.50 & 0.33 & 0.00 & 0.27 \\
\hline 2 & 0.00 & 0.50 & 0.17 & 0.00 & 0.17 & 0.50 & 0.17 & 0.50 & 0.25 \\
\hline 3 & 0.33 & 0.00 & 0.33 & 0.00 & 0.00 & 0.33 & & 0.00 & 0.17 \\
\hline 4 & 0.17 & 0.50 & 0.33 & 1.17 & 1.00 & 0.83 & 0.00 & 0.50 & 0.56 \\
\hline 5 & 0.33 & 0.33 & 0.17 & 0.17 & 0.50 & 0.33 & 0.50 & 0.83 & 0.40 \\
\hline 6 & 0.17 & 0.17 & 0.17 & 0.50 & 0.17 & 0.00 & 0.17 & 0.17 & 0.19 \\
\hline 7 & 0.17 & 0.17 & 0.00 & 0.50 & 0.00 & 0.00 & 0.17 & 0.17 & 0.15 \\
\hline 8 & 0.50 & 0.17 & 0.33 & 1.33 & 0.50 & 1.00 & 0.33 & 0.33 & 0.56 \\
\hline 9 & 0.17 & 0.17 & 0.17 & 0.33 & 0.17 & 0.33 & 0.17 & 0.00 & 0.19 \\
\hline 10 & 0.33 & 0.50 & 0.33 & 0.33 & 0.17 & 0.33 & 0.17 & 0.83 & 0.38 \\
\hline 11 & 0.00 & 0.83 & 0.67 & 0.17 & 0.17 & 0.33 & 0.17 & 0.00 & 0.29 \\
\hline 12 & 0.50 & 0.83 & 1.00 & 0.33 & 1.00 & 0.67 & 0.17 & 0.17 & 0.58 \\
\hline Mean & 0.25 & 0.35 & 0.32 & 0.44 & 0.35 & 0.43 & 0.22 & 0.29 & 0.33 \\
\hline SD & 0.17 & 0.29 & 0.27 & 0.42 & 0.34 & 0.30 & 0.13 & 0.31 & 0.28 \\
\hline
\end{tabular}

Absolute RPEDIFF = reported RPE - target RPE with sign dropped.

Values are the 3-week average of each subject's absolute RPEDIFF score for the listed lift and session. 
Resistance Exercise-Specific Rating of Perceived Exertion (RPE)

\begin{tabular}{c|c}
\hline Rating & Description of Perceived Exertion \\
\hline 10 & Maximum effort \\
\hline 9.5 & No further repetitions but could increase load \\
\hline 9 & 1 repetition remaining \\
\hline 8.5 & $1-2$ repetitions remaining \\
\hline 8 & 2 repetitions remaining \\
\hline 7.5 & $2-3$ repetitions remaining \\
\hline 7 & 3 repetitions remaining \\
\hline $5-6$ & $4-6$ repetitions remaining \\
\hline $3-4$ & Light effort \\
\hline $1-2$ & \\
\hline
\end{tabular}




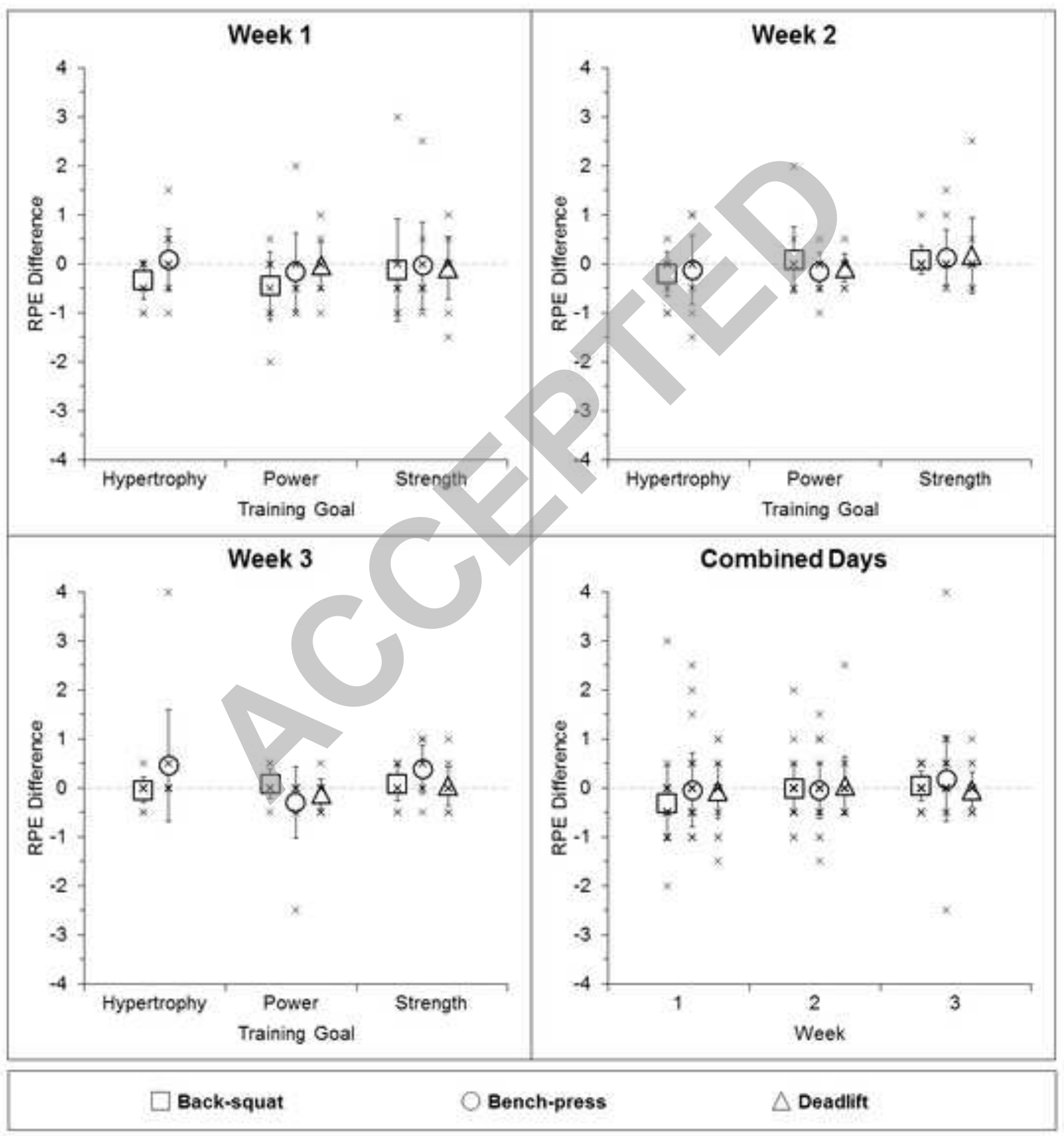

